\title{
Bladder tumours in children: An interesting case report of TCC with a partial inverted growth pattern
}

\author{
Davide Abed El Rahman ${ }^{1}$, Giuseppe Salvo ${ }^{2}$, Carlotta Palumbo ${ }^{3}$, Bernardo Rocco ${ }^{3}$, Francesco Rocco ${ }^{3}$ \\ ${ }^{1}$ U.O.C. di Urologia - Azienda Ospedaliera "G. Salvini”, Presidio di Rho, Rho (MI), Italy; \\ ${ }^{2}$ Dipartimento Universitario Materno-Infantile di Andrologia ed Urologia, Università degli Studi di Palermo, Italy; \\ ${ }^{3}$ Istituto di Urologia - Fondazione IRCCS - Ospedale Maggiore Policlinico "Ca Granda", Università degli Studi di Milano, Italy.
}

\begin{abstract}
Summary Bladder urothelial carcinoma is typically a disease of older individuals and rarely occurs below the age of 40 years.

There is debate and uncertainty in the literature regarding the clinicopathologic and prognostic characteristics of bladder urothelial neoplasms in younger patients compared with older patients, although no consistent age criteria have been used to define "younger" age group categories. We report on a 16 years old girl with transitional cell carcinoma of the bladder with a partial inverted growth pattern who presented with gross hematuria. Ultrasonography revealed a papillary lesion in the bladder; cystoscopic evaluation showed a $15 \mathrm{~mm}$ papillary lesion with a thick stalk located in the left bladder wall. Pathologic evaluation of the specimen was reported as "low grade transitional cell carcinoma of the bladder with a partial inverted growth pattern".
\end{abstract}

KEY WORDS: Bladder cancer; Young adults; Inverted papilloma; Pathology.

Submitted 24 April 2014; Accepted 30 June 2014

\section{INTRODUCTION}

Bladder urothelial cancer is predominately a disease of males and occurs mainly among the elderly adults (1), less than $1 \%$ of bladder urothelial cancer has been reported during the first four decades of life. Reported cases of transitional cell carcinoma of the bladder in the pediatric population are less than 150 .

In 1969 Javadpour and Mostofi studied the records of 10,000 patients with urothelial malignancy and found that just 40 patients were younger than 20 years old. Large series have described the characteristics of these tumours as low grade and seldom recurring (2).

Although the biologic behaviour and treatment of bladder cancer has been well studied, conflicting reports exist about clinical behavior and prognosis for patients under 40 years of age; whether younger patients have a better prognosis than their older counterparts has long been a subject of debate; indeed, some groups observed similar patterns of clinical behavior and prognosis for bladder cancer in young and older patients (3), whereas other investigators reported lower rates of disease recurrence and progression, and better survival, in younger patients (4). Moreover even if inverted papilloma of the urinary bladder is not uncommon in adults this is not the case in the pediatric population in which tumors of the urinary bladder are rare and more commonly of mesenchymal origin. Paschkis first described this lesion in 1927 and named it polypoid adenoma (5). Later, Potts and Hirst designated it as inverted papilloma (6). This lesion exhibits a polypoid configuration with an inverted papillomatous architecture lined by transitional epithelium. It also may have submucosal cyst formation and Brunn's nests so some authors refer to this lesion as Brunnian adenoma (7).

This lesion is rare in children, with the first case described in 1979 (8) and the youngest case described in a 11-year-old boy in 2000 (9). Furthermore, the exact biological behaviour of inverted papilloma of the urinary bladder remains uncertain.

We report an additional case of a low grade transitional cell carcinoma of the bladder with a partial inverted growth pattern in a 16 years old girl and emphasize the need for an intense and long-term follow-up of the pediatric population affected by bladder tumours (in its various histological types) to fully define its prognostic significance and potential biological behaviour in a near future.

\section{Case report}

We report the case of a non-smoking 16 years old girl without a clinically significant pathologic anamnesis who referred to another urology department for a double episode of asymptomatic macrohematuria in two months spontaneously resolved. The patient underwent an ultrasound scan of urinary bladder showing an endovescical papillary lesion of $15 \mathrm{~mm}$ located on the left side of bladder without associated ureterohydronephrosis. Cystoscopy demonstrated a $15 \mathrm{~mm}$ papillary lesion of the left bladder wall far from omolateral ureteral orifice; the lesion was connected to the bladder wall by a thin stalk. 
The patient underwent a transurethral resection of the bladder (TURB) and a 13 grams resection of newly formed tissue was performed.

The hystopathologic examination - performed by an expert urological pathologist - revealed a "low grade transitional cell carcinoma of the bladder with a partial inverted growth pattern in absence of subepithelial connective tissue infiltration".

After TURB the patient underwent a contrast enhanced computed tomography (TC) that showed a normal high urinary tract. Urologists gave indication for a "single shot" $40 \mathrm{ml}$ mytomicin C instillation, but the patient refused the treatment.

Two months later the patient contacted our department and, considering the rarity of the case and the extension of the neoplasm, we gave the indication of a " $2^{\text {nd }} l o o k$ " TURB that was performed after a few days.

Preoperative cystoscopy showed the area of previous resection interested by edema and partially covered by fibrin; there were not instead secure signs of persistent disease. We performed a deep resection of the described area and a separate collection of resection margins (medial, lateral, anterior and posterior). The biopsied areas were sent to the same expert pathologist who had done the previous diagnosis that revealed the absence of any suspicious area for recurrence.

The patient is now followed with cistoscopy and urinary citology every 3 months.

\section{Conclusions}

Younger patients with bladder cancer appear to have a more favourable prognosis, because they usually present with superficial stage and low-grade tumours. However, the risk of disease progression is the same, influenced by grade and stage at the time of presentation. Patients younger than 40 years old diagnosed with bladder cancer should be offered the same stage and grade appropriate management as older ones.

A diagnosis of bladder cancer should be considered in all patients with haematuria and bladder irritative symptoms, regardless of age.

\section{References}

1. Zhang ZL, Xiong YH, Li YH, et al. Reassessment of the predictive role of perivesical fat invasion in invasive bladder cancer prognosis in 151 Chinese patients. Chin Med J. 2011; 124:2915-2919.

2. Javadpour N, Mostofi FK. Primary epithelial tumors of the bladder in the first two decades of life. J Urol. 1969; 101:706-710.

3. Johnson DE, Hillis S. Carcinoma of the bladder in patients less than 40 years old. J Urol. 1978; 120:172-3.

4. Witjes JA, Debruyne FM. Bladder carcinoma in patients less than 40 years of age. Urol Int. 1989; 44:81-3.

5. Paschkis R. Uber Adenoma der Harnblase. Z Urol Chir. 1927; 21:315-325.

6. Potts IF, Hirst E. Inverted papilloma of the bladder. J Urol. 1963; 90:175-179.

7. Kim YH, Reiner L. Brunnian adenoma (inverted papilloma) of the urinary bladder: Report of a case. Hum Pathol. 1978; 9:229.

8. Lorentzen M, Rohr N. Urinary bladder tumours in children. Scand J Urol Nephrol. 1979; 13:323-327.

9. Isaac J, Lowicik A, et al. Inverted papilloma of the urinary bladder in children: case report and review of prognostic significance and biological potential behavior J Pediatr Surg. 35:1514-1516.

\section{Correspondence}

Davide Abed El Rahman, MD (Corresponding Author) davide.adel@libero.it

U.O.C. di Urologia - Azienda Ospedaliera "G. Salvini"

Presidio di Rho, viale Europa, 250 Rho (MI)

Giuseppe Salvo, MD

Dipartimento Universitario Materno-Infantile di Andrologia ed Urologia, Università degli Studi di Palermo, Palermo, Italy

Carlotta Palumbo, MD

Bernardo Rocco, MD

Francesco Rocco, MD

Istituto di Urologia - Fondazione IRCCS - Ospedale Maggiore

Policlinico "Ca Granda", Università degli Studi di Milano

Via delle Forze Armate, 260 - 20152 Milan, Italy 